\title{
Prevalence of myopia and its related risk factors among medical students in Saudi Arabia
}

\begin{abstract}
Background: Myopia or near-sightedness is the most frequent cause of vision deterioration with growing burden worldwide. Although myopia is hereditary, nearsightedness is tremendously increasing due to endlessly focusing on close objects. Therefore, the objective of the present study was to assess the burden of nearsightedness and its related risk factors among medical students in the Kingdom of Saudi Arabia (KSA).
\end{abstract}

Methodology: This study involved 504 medical students (346 were males and 158 were females) selected from two medical colleges from (Hail University and Prince Sattam University). Data were collected using a purposeful questionnaire.

Results: Of the 504 participants, 454 (90\%) have responded of whom 243 (53.5\%) were found with nearsightedness phenomenon. Of the 243 students with myopia, 159/243(65.4\%) males and $84 / 243(34.6 \%)$ were females. Out of the 248 respondents to question of vision's aid usage, $185 / 248(74.6 \%)$ were using glasses, $25 / 248(10 \%)$ were using contact lenses, and the remaining $38 / 248(15.4 \%)$ were none aids users.

Conclusion: Myopia is prevalent among medical students which might be due to the visual stress that associated with doing detailed work, such as reading, using a computer or a mobile phone.

Keywords: myopia, nearsightedness, Saudi Arabia
Volume 6 Issue I - 2017

Rakan Mosa Algorinees, Naif Theeb Alqahtani, Abdulhkam Moafak Aljarbou, Reem Saleh AlShammari, Ali Ghannam Alrashidi, Bandar Turqi Alshammari, Hussain Gadelkarim Ahmed

University of Hail, Saudi Arabia

Correspondence: Hussain Gadelkarim Ahmed, College of Medicine, University of Hail, Saudi Arabia, Tel +966502609300, Email hussaingad1972@yahoo.com

Received: December 06, 2016 | Published: January 18, 2017
Abbreviations: KSA, kingdom of Saudi Arabia; SE, spherical equivalent; CrI, credible intervals; OK, orthokeratology; SV, single vision

\section{Introduction}

Myopia, the utmost frequent form of refractive error, is a complex characteristic comprising both environmental and genetic factors. ${ }^{1}$ Myopia is a reciprocal risk factor for vision loss, and the leading cause of distance vision impairment worldwide. Discrete studies reported variations in the prevalence of myopia between regions and ethnic groups. In a study, which incorporated data from 145 studies covering 2.1 million participants, 1406 million people were detected with myopia representing $22.9 \%$ of the world's population. ${ }^{2}$ In the kingdom of Saudi Arabia (KSA), the prevalence of refractive error among children was up to $18.5 \%{ }^{3}$ In study from Saudi Arabia investigated 2246 Saudi primary school children aged 6 to 14 years, Myopia was the most commonly encountered refractive error among both genders $\left(65.7 \%\right.$ of the total errors encountered. ${ }^{4}$

Several studies have attempted to interpret the etiology of myopia, but the concrete etiology of myopia is still obscure. Environmental factors play an essential role in the development of myopia. The influence of genetic and environmental factors interaction on the etiology of myopia is yet debatable with varying findings in various studies. ${ }^{1}$ Several causes have been recognized for having potential associations with risks for the development of myopia, near-work load, occupation, education, income, outdoor activities, lens opacity, ocular dimensions, parental myopia, gender and ethnicity. ${ }^{5-8}$ Nearwork activities, such as, writing, reading, computer or smart phone usage, and playing video games, have been proposed to be responsible for the notable rise in the prevalence of myopia. ${ }^{9}$ Some studies revealed increased prevalence of myopia among those with higher education levels, better housing, higher income and occupations associated with near work. ${ }^{10}$ A number of epidemiological studies indicated that more time spent outdoors might be associated with lower prevalence of myopia. ${ }^{11}$ Myopia seems to be more often seen in children with myopic parents. It was reported that the proportions of myopia were $6.3 \%$ in schoolchildren aged $13.7 \pm 0.5$ years whose parents are both emmetropic, $18.2 \%$ in children with one myopic parent, and $32.9 \%$ in children who's both parents are myopic. ${ }^{12}$

It has also been well-established that myopia, particularly high myopia, might be affected by compromised quality of life due to a number of influences including psychological, functional, and financial factors cosmetic. ${ }^{13}$ The prevalence of myopia is alarmingly increasing in KSA. Several environmental risk factors related to lifestyle and socioeconomic status may possibly be associated with these changes. Evidence has also been generated in regard to the theory that myopia is the result of a complicated interaction between genetic predisposition and environmental factors. Measurement of these factors in different population settings is very important for better prevention and control strategies. Therefore, the objective of the present study was to determine the epidemiologic patterns of myopia among university medical students.

\section{Materials and methods}

In this cross sectional study, collected data regarding nearsightedness was obtained from 504 Saudi medical student volunteers living in two cities of the Kingdom of Saudi Arabia (KSA) including; Hail (Northern KSA), and Riyadh (Capital KSA). Out of the 504 medical students, $346(68.7 \%)$ were males and $158(31.3 \%)$ 
were females) selected from two medical colleges, College of Medicine, University of Hail and College of Medicine (in Hail city), Prince Sattam University (in Riyadh city). A purposeful questionnaire was designed and used for collection of the required data regarding vision status and indications of genetics and environmental factors. The following information was obtained from each participant: age; sex; current vision status (-decreased vision- increased visionnormal vision); family member with myopia; children with myopia; outdoor activities; reading and writing activities; computer, iPad and smart phone usage; myopia associated eye (right, left or both); degree of vision status according to Snellen-Chart; use of vision aids (glasses, contact lenses or others); and any information regarding other previous or current eye disorders. Myopia was detected using cycloplegic refraction. Diagnosis of myopia was confirmed when a spherical equivalent (SE) of -0.50 diopter (D) or worse in one or both eyes. The severity of nearsightedness is categorized according to: Mild myopia: -0.50 to $-2.99 \mathrm{D}$; Moderate myopia: -3.00 to $-6.00 \mathrm{D}$; High myopia: greater than $-6.00 \mathrm{D}$.

\section{Data analysis}

Data was analyzed using SPSS software program (version 16). Conferring percentage and $95 \%$ confidence interval were used to define the prevalence and distribution of the different variables. Pearson Chi-squared test was used for qualitative data. A $P$-value of $<5 \%$ was statistically significant.

\section{Results}

This study screened 504 medical students for the presence of myopia, their ages ranging from 18 to 27 years old with a mean age of 21 years. Of the 504 participants, 454/504(90\%) were examined for the presence of myopia or hypermetropia. Of the 454examined students, myopia was detected in 243/454(53.5\%) of whom 159/243(65.4\%) males and 84/243(34.6\%) were females. Hypermetropia was defined in 9/454(2\%) individuals of whom 7 were males and 2 were females, as indicated in Table 1.

Table I Distribution of the vision status by sex, age and family history

\begin{tabular}{|c|c|c|c|c|c|}
\hline Variable & Category & Myopia & Hypermetropia & Normal & Total \\
\hline \multicolumn{6}{|l|}{ Sex } \\
\hline & Males & 159 & 7 & 141 & 307 \\
\hline & Females & 84 & 2 & 61 & 147 \\
\hline & Total & 243 & 9 & 202 & 454 \\
\hline \multicolumn{6}{|l|}{ Age } \\
\hline & $\leq 20$ years & 77 & 3 & 83 & 162 \\
\hline & $21-23 y r s$ & 128 & 6 & 100 & 234 \\
\hline & $\geq 24 \mathrm{yrs}$ & 38 & 0 & 17 & 54 \\
\hline & Total & 243 & 9 & 200 & 452 \\
\hline \multicolumn{6}{|c|}{ Family history of myopia } \\
\hline & Present & 209 & 3 & 129 & 341 \\
\hline & Absent & 26 & 6 & 73 & 105 \\
\hline & Total & 235 & 9 & 202 & 446 \\
\hline
\end{tabular}

Myopia was found in $77 / 243(31.7 \%)$, I28/243(52.7\%) and 38/243(I5.6\%) of the age groups, $\leq 20$ years, $21-23$, and $\geq 24$ years, respectively. Hypermetropia was found in $3 / 9(33.3 \%)$ of the age group $\leq 20$ and in $6 / 9(66.7 \%)$ of the age range $2 \mathrm{I}-23$ years, as shown in Table I

When comparing myopia categories within each variable, for sex, myopia was more common among female group compared to males. For age, myopia was more elderly age groups. Family history was very high among those with myopia as indicated in Figure 1. The great majority of the cases were with moderate myopia regardless of the affected eye. Of the 137 cases of myopia with affected right eye,
$31 / 137(22.6 \%), 92 / 137(67.2 \%)$ and 14/137(10.2\%) were identified with mild, moderate and high myopia, respectively. Of the 137 cases of myopia with affected left eye, 40/137 (29.2\%), 76/137(55.5\%) and $21 / 137(15.3 \%)$ were identified with mild, moderate and high myopia, respectively. 


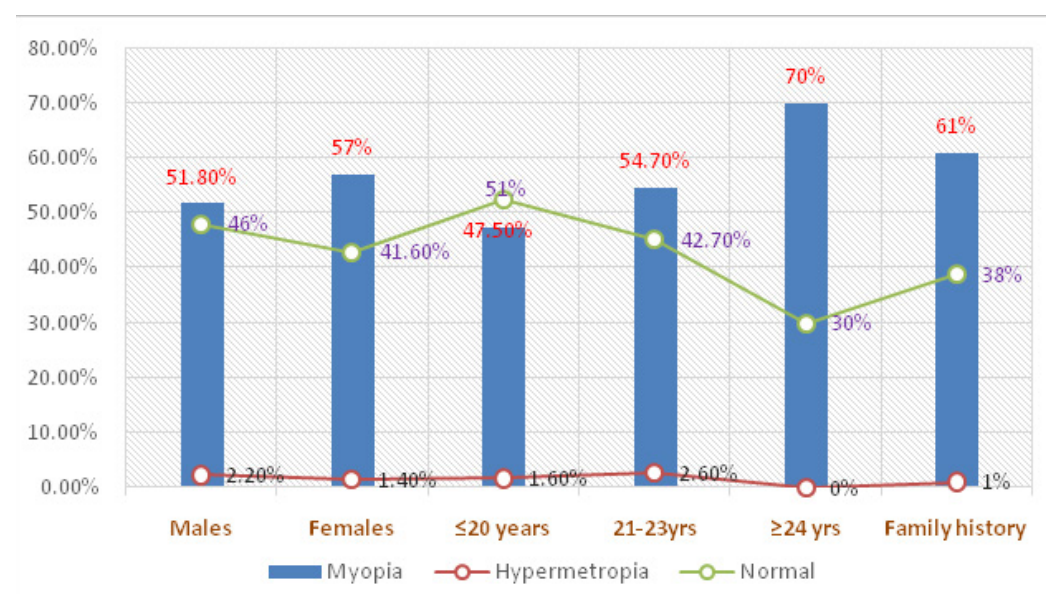

Figure I Description of the vision status by sex, age and family history within each group.

Of the 83 cases of myopia with affected both eyes, $27 / 83(32.5 \%)$, $43 / 83(51.8 \%)$ and $13 / 83(15.7 \%)$ were identified with mild, moderate and high myopia, correspondingly, as indicated in Table 2, Figure 2. Of the 231 respondents to vision aids' usage, 174/231(75.3\%) were using glasses, and using contact lenses $25 / 174(10.8 \%)$ and the remaining $32 / 231(13.9 \%)$ were currently without aids. In regard to the age of wearing the vision aids, 66/204(32.2\%), 61/204(30\%),

Table 2 Distribution of the affected eye (s) by measurements of myopia
$61 / 204(30 \%)$ and $16 / 204(7.8 \%)$ wore the vision aids when they were at the age of $\leq 15$ years, $16-18,19-21$ and $\geq 22$ years, respectively. The distribution of males and females is relatively similar in regard to the total participants in each category, as indicated in Table 3 . When calculating the percentage within each sex group, it shows little variability as shown in Figure 3.

\begin{tabular}{llll}
\hline Variable & Right eye & Left eye & Both eyes \\
\hline Diopters & & & \\
-0.5 to-0.99 D & 3 & 5 & 3 \\
-1.00 to-1.99 D & 20 & 19 & 16 \\
-2.00 to-2.99 D & 8 & 16 & 8 \\
-3.00 to-3.99 D & 24 & 21 & 16 \\
-4.00 to-4.99 D & 35 & 27 & 14 \\
-5.00 to-5.99 D & 33 & 28 & 13 \\
-6.00 and more D & 14 & 21 & 13 \\
Total & 137 & 137 & 83 \\
\hline Mild Myopia & Moderate Myopia & High Myopia
\end{tabular}

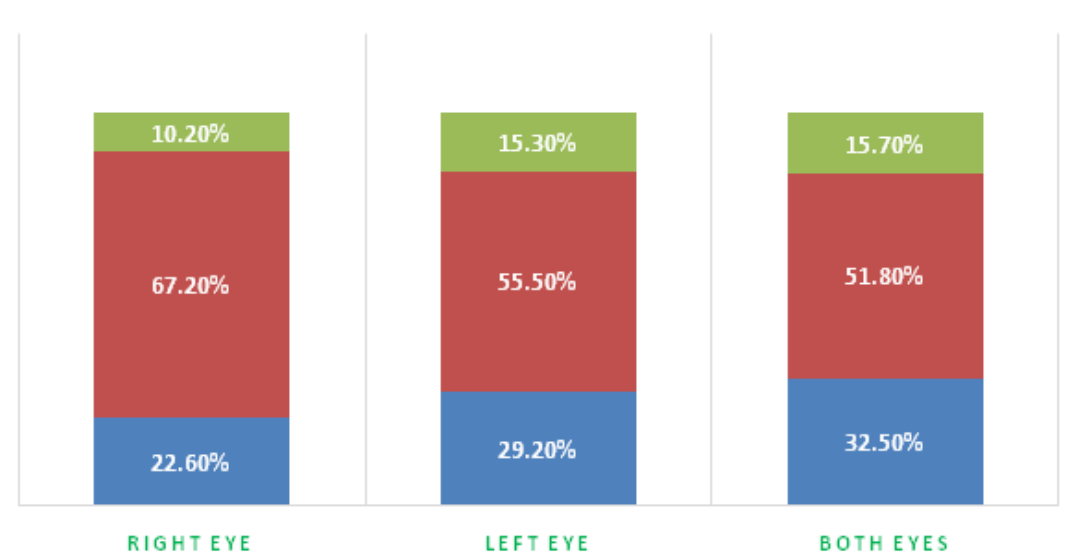

Figure 2 Description of the affected eye (s) by degree of myopia. 
Table 3 Distribution of the myopia cases by sex and vision aids usage

\begin{tabular}{|c|c|c|c|c|}
\hline Variable & Category & Males & Females & Tota \\
\hline \multicolumn{5}{|c|}{ Age of wearing vision aids } \\
\hline & $\leq 15$ years & 43 & 23 & 66 \\
\hline & $16-18$ & 38 & 23 & 61 \\
\hline & $|9-2|$ & 37 & 24 & 61 \\
\hline & $\geq 22$ years & 14 & 2 & 16 \\
\hline & Total & 132 & 72 & 204 \\
\hline
\end{tabular}

Period of wearing vision aids

$\begin{array}{llll}\text { All day time } & 65 & 32 & 97 \\ \text { Most day time } & 22 & 8 & 30 \\ \text { Occasionally } & 42 & 33 & 75 \\ \text { Total } & 129 & 73 & 202\end{array}$

Period of changing vision aids

$\begin{array}{lccc}\leq 1 \text { year } & 93 & 54 & 147 \\ >\text { I year } & 35 & 19 & 54 \\ \text { Total } & 128 & 73 & 201\end{array}$

\section{Cause of aid change}

\begin{tabular}{lccc} 
Uneasy eyes & 45 & 37 & 82 \\
Better frame of lenses & 82 & 34 & 116 \\
Total & 127 & 71 & 198 \\
\hline
\end{tabular}

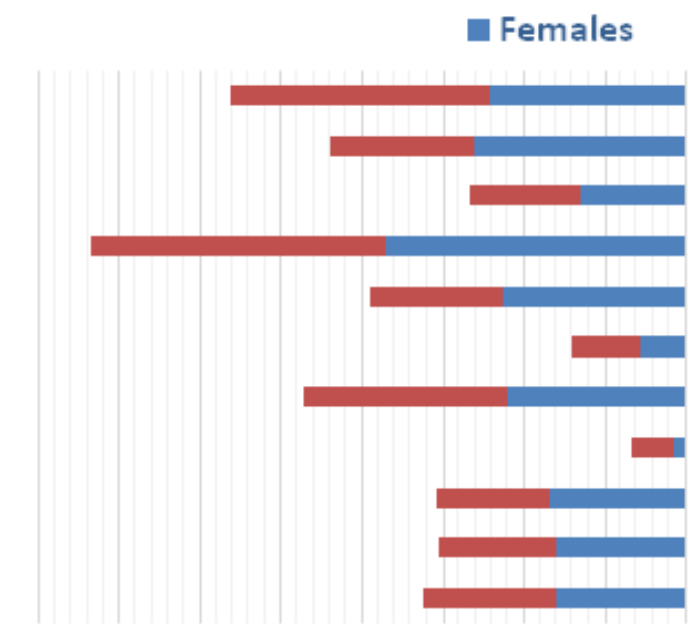

Males

Change because better frame or lenese

Change because (uneasy eyes)

Changing within $>1$ year

Changing within $\leq 1$ year

Occasionally

Most day

Wearing all day

$\geq 22$ years

19-21yrs

$16-18 y r s$

Start wearing at age $\leq 15$ years

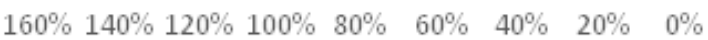

Figure 3 Description of the myopia cases by sex and vision aids usage. 
For wearing of vision aids, 97/202(48\%), 30/202(15\%) and $75 / 202(37 \%)$ individuals were found to wear the visions' aids, all day, most of the day and occasionally, in this order, as indicated in Table 3 . When calculating the percentages with each sex, the majority of males wearing vision aids all day, hence, most females wear vision aids occasionally, as shown in Figure 3. For period of changing vision aids, $147 / 201(73 \%)$ people used to change their vision aids within one year or less and 54/201(27\%) their vision aid for periods of more than one year. Most males and females change within one year or less, as shown in Table 3, Figure 3. For the reason of changing the vision aids, $82 / 198(41 \%)$ were changing because they with feeling uneasy eyes and 116/198(59\%) were changing because they got better frame or lenses. Most males changing because of better frame or lenses, whereas, most females changing because of uneasy eyes feeling, as shown in Table 3, Figure 3.
Table 4 summarizes the distribution of the myopia cases by sex and vision habits (nearsightedness work). Of the 240 persons using smart devices (computers, iPad and smart phones), 103/240(43\%), $104 / 240(43.3 \%)$ and 33/240(13.7\%) were using these devices for $\leq 5$ hours, $6-10$ hours and $\geq 11$ hours, correspondingly. Most males used to use for 5 hours or more, whereas, the majority of females use for 6-10 hours. Of the 231 individuals who read books, 197/231(85\%) were reading for $\leq 5$ hours per day and $34 / 231(15 \%)$ were reading for more than 5 hours per day. The majority of males and females used to read for 5 hours or more. Of the 220 individuals enabled to estimated their reading papers, 49/220(22.3\%), 39/220(17.7\%) and $132 / 220(60 \%)$ were found using devices, reading books and reading papers, respectively. The great percentages of males and females used to read 11 papers or more per day, as indicated in Table 4, Figure 4.

Table 4 Distribution of the myopia cases by sex and vision habits (nearsightedness work)

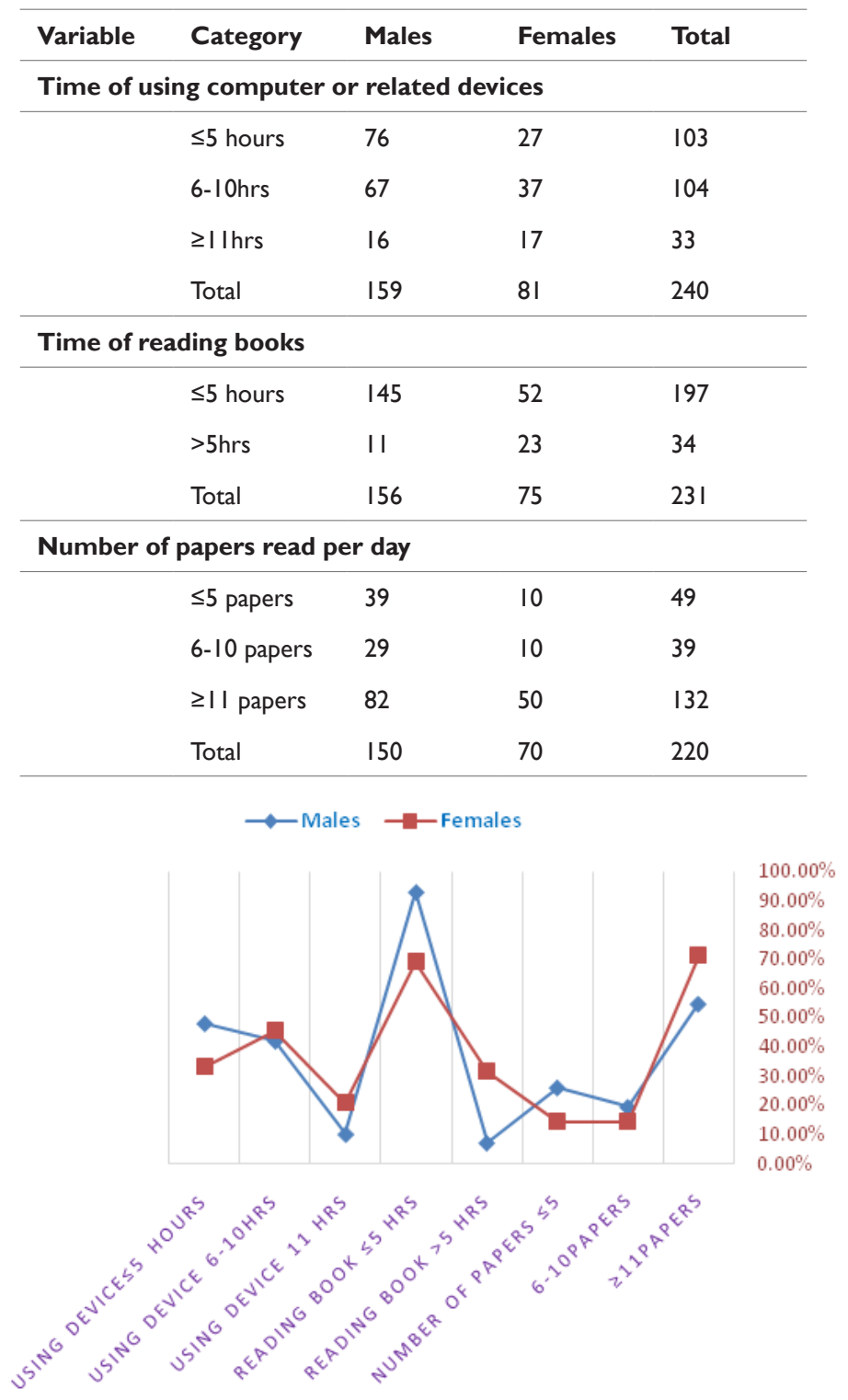

Figure 4 Description of the myopia cases by vision habits (nearsightedness work) and proportions with females and males. 


\section{Discussion}

Myopia is a most frequent cause of vision impairment with increasing prevalence worldwide. There is a wide variation in the prevalence of myopia between regions and ethnic groups. The present study has randomly selected 504 medical students from a total of about 1200 medical students who were currently enrolled in two medical schools. Of the studied group, the prevalence of myopia was $53.5 \%$ and the prevalence rates of males and females were $65.4 \%$ and $34.6 \%$, respectively. In a review to measure the global disparity in childhood myopia prevalence over time considering demographic factors; there is a rise in myopia prevalence rates with age varied by ethnicity. East Asians exhibited the highest prevalence, attaining 69\% (95\% credible intervals (CrI) $61 \%$ to $77 \%$ ) at 15 years of age. ${ }^{14}$ Studies on school children in China revealed myopia prevalence rates ranging from $20 \%$ in younger children, and $80 \%$ in 17 -year-old students. ${ }^{15,16}$

Studies from KSA in this context are very few and most of them dealt with children. In a study conducted to identify the prevalence and pattern of refractive errors among the school-entrant children, the prevalence of myopia $(2.5 \%, 95 \% \mathrm{CI} 1.7: 3.3 \%) .{ }^{17}$ Another study from KSA, has examined 2246 Saudi primary school children aged 6 to 14 years of both genders. The study found that myopia was the most frequently met refractive error among both genders $(65.7 \%$ of the total errors encountered). ${ }^{4}$ Another cross-sectional survey conducted in KSA included 21 primary schools with of 5176 children (mean age $9.5 \pm 1.8$ years), the inclusive prevalence of RE was $18.6 \%$, and astigmatism and myopia were the most common refractive error. ${ }^{3}$ All these related studies have screened for myopia among younger children which may reduce the attribute ability of myopia occurrence. In the present study, the highest percentage of cases with myopia were seen among those at 24 years old or older. It was reported that the myopia rate increased significantly with age. ${ }^{18}$

On the other hand, the enrolled females in the present study were far less than males' number, which might not give a comparable percentage. Therefore, when counting the percentage from each sex group, different percentages can be noticed as indicated in the results. Within female's group, the percentage of myopia was greater than within the male's group. Such findings were previously reported in some studies. ${ }^{19}$ In the current study $61 \%$ of those with myopia have a family history of myopia. Compared with children without myopic parents, those with one myopic parent are twice as expected to be myopic, and those with two parents myopic are three times more expected to be myopic themselves. ${ }^{20}$ About $60.7 \%$ of the cases of myopia were affected in both eyes of which $32.5 \%, 51.8 \%$ and $15.7 \%$ were detected with mild, moderate and high myopia, respectively. Luckily those with high myopia represent only small proportion in the present study and most frequently in the left eye than right eye.

Approximately $75.3 \%$ of myopia cases were using glasses, and using contact lenses $10.8 \%$ and the remaining $13.9 \%$ were currently without aids. What is interesting these results, that $13.9 \%$ myopia cases were detected during this survey. The use of both spectacles and contact lenses offers acceptable visual functioning for everyday activities in young people suffering from mild to moderate myopia. Nevertheless, there is an advantage of spectacles wear in personal satisfaction when compared to contact lenses. ${ }^{21}$ In study comparing vision-related quality-of-life measures between children wearing Orthokeratology (OK) contact lenses and distance single-vision (SV) spectacles. A significant enhancement in vision-related quality of life and satisfactoriness with $\mathrm{OK}$ contact lenses is a motivation to engage in its usage for the control of myopia in children. ${ }^{22}$ The relatively low incidence of contrary actions and discontinuations with $\mathrm{OK}$ is encouraging for the correction of myopia in children with OK contact lenses. ${ }^{23}$ Moreover, it was found that both $\mathrm{OK}$ and dual focus soft contact lenses are effective strategies for targeting myopia evolution in the clinic. No significant difference in the effectiveness of the two measures in this respect, and accordingly there are very few barriers for any contact lens practitioner to be actively endorsing myopia control treatment to patients at risk. ${ }^{24}$

The great majority of individuals had worn the vision aids before the age of 18 years old. However, about $37 \%$ of the cases with myopia used to wear the vision aids occasionally and around $15 \%$ were the vision aids discontinuously with the day. The influence of continuous wearing of vision aids on the progress or regress of myopia is an issue that need more investigations. Moreover, there was reasonable number of cases used to change their vision aids after more than one year of use. How far old vision aids affect the process of myopia is another concerned issue, but what is interesting that a great majority of individuals changing their vision aids for leisure reasons (better frame or lenses). However, these might indirectly indicate the socioeconomic of the studied section, which represents as a risk factor for progress of myopia. This in addition to the fact that most of these students have limited outdoor activities, because they are always busy with their studying. Factors such as time of using devices, times of books reading and number of paper did not seem to influence the degree of myopia in the present study. The limitations of the present study, include its cross-sectional setting, it's targeting of medical students who might be more suitable for developing myopia due intensive reading and writing, and the relatively lower sample size in such type of studies.

\section{Conclusion}

The findings of this study indicate that there is a high prevalence of myopia among medical students in Saudi Arabia. Life style amendment, such as more out-door activities and limited nearwork activity, vision check-up after medical college enrolment may decrease the increasingly high prevalence of myopia among medical students in KSA.

\section{Ethical consent}

Each participant was asked to sign a written ethical consent during the questionnaire's interview. The informed ethical consent form was designed and approved by the ethical committee of the College of Medicine (University of Hail, KSA) Research Board.

\section{Funding}

None.

\section{Acknowledgments}

None.

\section{Conflicts of interest}

The authors declare that there was no conflict of interest.

\section{References}

1. Pan CW, Ramamurthy D, Saw SM. Worldwide prevalence and risk factors for myopia. Ophthalmic Physiol Opt. 2012;32(1):3-16. 
2. Holden BA, Fricke TR, Wilson DA, et al. Global Prevalence of Myopia and High Myopia and Temporal Trends from 2000 through 2050 Ophthalmology. 2016;123(5):1036-1042.

3. Aldebasi YH. Prevalence of correctable visual impairment in primary school children in Qassim Province, Saudi Arabia. J Optom. 2014;7(3):168-176.

4. Al Wadaani FA, Amin TT, Ali A, et al. Prevalence and Pattern of Refractive Errors among Primary School Children in Al Hassa, Saudi Arabia. Glob J Health Sci. 2013;5(1):125-134.

5. Wong TY, Foster PJ, Hee J, et al. Prevalence and risk factors for refractive errors in adult Chinese in Singapore. Invest Ophthalmol Vis Sci. 2000;41(9):2486-2494.

6. Wong TY, Foster PJ, Johnson GJ, et al. The relationship between ocular dimensions and refraction with adult stature:the TanjongPagar Survey. Invest Ophthalmol Vis Sci. 2001;42(6):1237-1242.

7. Morgan A, Young R, Narankhand B, et al. Prevalence rate of myopia in schoolchildren in rural Mongolia. Optom Vis Sci. 2006;83(1):53-56.

8. Ahmed I, Mian S, Mudasir S, et al. Prevalence of myopia in students of srinagar city of kashmir, India. Int J Health Sci (Qassim). 2008;2(1):7781 .

9. Ip JM, Huynh SC, Robaei D, et al. Ethnic differences in the impact of parental myopia:findings from a population-based study of 12-year-old Australian children. Invest Ophthalmol Vis Sci. 2007;48(6):2520-2528.

10. Wong TY, Foster PJ, Johnson GJ, et al. Refractive errors, axial ocular dimensions, and age-related cataracts:the TanjongPagar survey. Invest Ophthalmol Vis Sci. 2003;44(4):1479-1485.

11. Sherwin JC, Reacher MH, Keogh RH, et al. The association between time spent outdoors and myopia in children and adolescents:a systematic review and meta-analysis. Ophthalmology. 2012;119(10):2141-2151.

12. Mutti DO, Mitchell GL, Moeschberger ML, et al. Parental myopia, near work, school achievement, and children's refractive error. Invest Ophthalmol Vis Sci. 2002;43(12):3633-3640.

13. Foster PJ, Jiang Y. Epidemiology of myopia. Eye (Lond). 2014;28(2):202208.
14. Rudnicka AR, Kapetanakis VV, Wathern AK, et al. Global variations and time trends in the prevalence of childhood myopia, a systematic review and quantitative meta-analysis:implications for aetiology and early prevention. Br J Ophthalmol. 2016;100(7):882-890.

15. Pi LH, Chen L, Liu Q, et al. Refractive status and prevalence of refractive errors in suburban school-age children. Int J Med Sci. 2010;7(6):342353.

16. You QS, Wu LJ, Duan JL, et al. Prevalence of myopia in school children in greater Beijing; the Beijing Childhood Eye Study. Acta Ophthalmol. 2014;92(5):e398-406.

17. Al-Rowaily MA. Prevalence of refractive errors among pre-school children at King Abdulaziz Medical City, Riyadh, Saudi Arabia. Saudi J Ophthalmol. 2010;24(2):45-48.

18. Chebil A, Jedidi L, Chaker N, et al. Epidemiologic study of myopia in a population of schoolchildren in Tunisia. Tunis Med. 2016;94(3):216-220.

19. Mehari ZA, Yimer AW. Prevalence of refractive errors among schoolchildren in rural central Ethiopia. Clin Exp Optom. 2013;96(1):6569

20. Xiang F, He M, Morgan IG. The impact of parental myopia on myopia in Chinese children:population-based evidence. Optom Vis Sci. 2012;89(10):1487-1496.

21. Kanonidou E, Chatziralli IP, Konidaris V, et al. A comparative study of visual function of young myopic adults wearing contact lenses vs. spectacles. Cont Lens Anterior Eye. 2012;35(5):196-198.

22. Santodomingo-Rubido J, Villa-CollarC, Gilmartin B, et al. Myopia control with orthokeratology contact lenses in Spain:a comparison of visionrelated quality-of-life measures between orthokeratology contact lenses and single-vision spectacles. Eye Contact Lens. 2013;39(2):153_ 157.

23. Santodomingo-Rubido J, Villa-Collar C, Gilmartin B, et al. Orthokeratology vs. spectacles: adverse events and discontinuations. Optom Vis Sci. 2012;89(8):1133-1139.

24. Turnbull PR, Munro OJ, Phillips JR. Contact Lens Methods for Clinical Myopia Control. Optom Vis Sci. 2016;93(9):1120-1126. 